\title{
Influence of Shape Anisotropy on Magnetization Dynamics Driven by Spin Hall Effect
}

\author{
X. G. Li, ${ }^{1}$ Z. J. Liu, ${ }^{1}$ X. Y. Xie, ${ }^{1}$ A. G. Kang, ${ }^{1}$ and W. N. Fu ${ }^{2}$ \\ ${ }^{1}$ College of Physics and Optoelectronics, Taiyuan University of Technology, Taiyuan, China \\ ${ }^{2}$ Department of Electrical Engineering, The Hong Kong Polytechnic University, Kowloon, Hong Kong \\ Correspondence should be addressed to Z. J. Liu; eleliuzj@126.com
}

Received 3 June 2016; Revised 13 October 2016; Accepted 1 November 2016

Academic Editor: Santiago Garcia-Granda

Copyright (c) 2016 X. G. Li et al. This is an open access article distributed under the Creative Commons Attribution License, which permits unrestricted use, distribution, and reproduction in any medium, provided the original work is properly cited.

\begin{abstract}
As the lateral dimension of spin Hall effect based magnetic random-access memory (SHE-RAM) devices is scaled down, shape anisotropy has varied influence on both the magnetic field and the current-driven switching characteristics. In this paper, we study such influences on elliptic film nanomagnets and theoretically investigate the switching characteristics for SHE-RAM element with in-plane magnetization. The analytical expressions for critical current density are presented and the results are compared with those obtained from macrospin and micromagnetic simulation. It is found that the key performance indicators for in-plane SHE-RAM, including thermal stability and spin torque efficiency, are highly geometry dependent and can be effectively improved by geometric design.
\end{abstract}

\section{Introduction}

The effect of spin-transfer torque [1-3] has been investigated extensively in the last decade due to its potential applications in spin-transfer torque magnetic random-access memory (STT-RAM) [4-7], nanoscale microwave generators [7-10], and spin based logic devices [11]. The functional element utilizing spin-transfer torque is commonly a magnetic tunnel junction (MTJ) with a magnetic free layer and reference layer separated by a tunneling barrier. For STT-RAM, the bit is stored by the relative orientation of magnetization of free layer and reference layer, and the polarized current is used to change its state between parallel (P) and antiparallel (AP). In order to balance the demands for low threshold current, high thermal stability, and small element size, MTJ stacks with different materials and structures have been proposed. In the last few years, MTJ with perpendicular magnetization has attracted much attention since the strong interfacial magnetic anisotropy in $\mathrm{CoFeB} / \mathrm{MgO}$ bilayer reduces the switching current density by canceling out the demagnetization field [1214]. However, as the element dimension keeps scaling down, time dependent dielectric breakdown (TDDB) becomes a critical issue restraining the performance of perpendicular
MTJ [15]. More recently, a three-terminal MTJ utilizing spin Hall effect (SHE) [16] has been demonstrated to be an effective solution $[4,17,18]$. Pure spin current, due to spin orbit interaction, can be generated by applying a current to a heavy metal $(\beta-\mathrm{W}[19,20], \beta-\mathrm{Ta}[21]$, and Pt [22]) adjacent to the free layer of MTJ.

Apparently, the SHE-RAM allows for further scaling down of the nonvolatile memory element. However, there is a critical issue from the scaling perspective, that is, the influence of shape anisotropy on switching characteristics, which needs to be carefully examined. In previous macrospin based researches, simplified expressions of the shape anisotropy were often used with the assumption that the area of free layer is large compared with its thickness. However, as the element dimension is scaled down, this simplification frequently becomes a nontrivial source of error when studying the switching current and thermal stability of STT-RAM elements [23-26]. The problem can be addressed by either micromagnetic modeling or accurate demagnetizing factors for macrospin model.

In this paper, we introduce the analytic demagnetizing factors for elliptic film nanomagnets and theoretically investigate the geometric dependent critical current density 


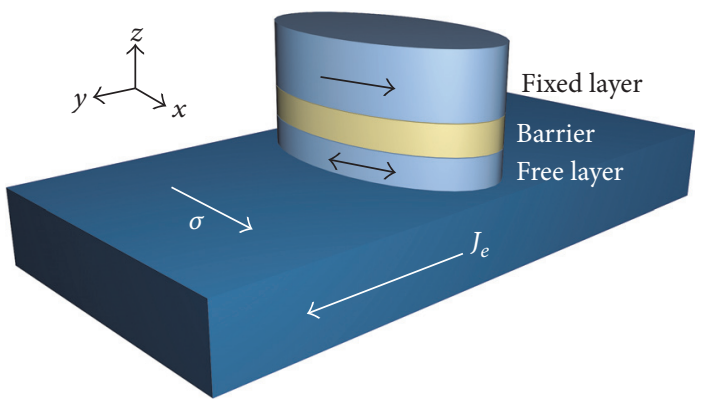

(a)

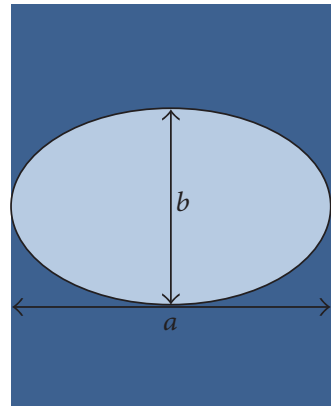

(b)

FIGURE 1: The sketch of in-plane SHE-RAM element.

for SHE-RAM with in-plane magnetization. We present the analytic expressions for both current densities leading to magnetization instability and switching. The analytic results are compared with macrospin and micromagnetic numerical simulation, and good consistency is found below a critical size. We further analyze the geometric dependent thermal stability and spin torque efficiency in room temperature, and it is found that these key performance indicators can be effectively improved by geometric design.

The remainder of this paper is organized as follows. Section 2 gives the theoretical model and analytic equations for demagnetizing factors and critical current density. Section 3 presents the dependence of zero temperature critical current density on free layer geometry and the comparisons between analytic expression, macrospin, and micromagnetic simulation. Section 4 introduces thermal fluctuation by stochastic LLG equation, and the element characteristics concerning thermal fluctuation are investigated. Section 5 summarizes the paper and draws the conclusion. SI units are used for equations and results in this paper.

\section{Model Detail}

2.1. LLGS Equation. Figure 1 is a sketch of the in-plane SHERAM element. The short axis lies in $y$ direction with length $b$. The long axis of the free layer lies in $x$ direction with length $a$, which is also the width of the heavy metal layer, as shown in Figure 1(b).

The magnetization dynamics of the in-plane MTJ driven by SHE polarized current are similar to that of a conventional STT-RAM and can be described by the Landau-LifshitzGilbert equation with an SHE spin torque term [27] as follows:

$$
\begin{aligned}
\frac{d \mathbf{m}}{d t}= & -\gamma \mathbf{m} \times\left(\mathbf{H}_{\text {eff }}+\mathbf{H}_{\text {the }}\right)+\alpha \mathbf{m} \times \frac{d \mathbf{m}}{d t}+a_{J} \mathbf{m} \\
& \times(\mathbf{m} \times \boldsymbol{\sigma}),
\end{aligned}
$$

where $\mathbf{m}$ and $\boldsymbol{\sigma}$ are the normalized magnetization of the free layer and spin polarization vector, respectively, $\gamma=$ $1.76 \times 10^{11} \mathrm{~T}^{-1} \mathrm{~s}^{-1}$ is the gyromagnetic ratio, $\alpha$ is the Gilbert damping constant, and $a_{J}$ is the spin torque strength which can be expressed as [28]

$$
a_{J}=\frac{\gamma \hbar J_{e} \theta_{\mathrm{SH}}^{\mathrm{eff}}}{2 \mu_{0} e t_{f} M_{s}}
$$

where $\hbar$ is the reduced Planck constant, $J_{e}$ is the charge current density applied to the heavy metal layer in the $y$ direction, $e$ is the electron charge, $\mu_{0}$ is the permeability of vacuum, $\theta_{\mathrm{SH}}^{\mathrm{eff}}$ is the material dependent effective spin Hall angle, and $t_{f}$ is the thickness of the free layer. In our latter discussion, we assume $\alpha=0.01, M_{s}=1 \times 10^{6} \mathrm{~A} / \mathrm{m}$, and $\theta_{\mathrm{SH}}^{\text {eff }}=$ 0.32 to simulate the CoFeB-based MTJ adjacent to an $8 \mathrm{~nm} \beta$ $\mathrm{W}$ thin film [20]. $\mathbf{H}_{\text {the }}$ is the stochastic field related to thermal fluctuation. Based on the framework of Brown [29], it can be expressed as follows:

$$
\begin{aligned}
\left\langle\mathbf{H}_{\text {the }}\right\rangle & =0, \\
\left\langle\mathbf{H}_{\text {the }, i}\left(t_{1}\right) \mathbf{H}_{\text {the }, j}\left(t_{2}\right)\right\rangle & =\frac{2 k_{b} T \alpha}{\mu_{0} \gamma M_{s} V} \delta_{i j} \delta\left(t_{1}-t_{2}\right),
\end{aligned}
$$

where $i, j=1,2$, and 3 and $\mathbf{H}_{\text {the }, i}$ refers to the Cartesian component of the stochastic field. $k_{b}$ is the Boltzmann constant, $T$ is the temperature in kelvin, and $V$ is the volume of the free layer. $\mathbf{H}_{\text {eff }}$ represents the effective field. Since bulk anisotropy is ruled out, the energy density of the free layer with a zero applied field is

$$
E_{\text {total }}=E_{\text {demag }}=\frac{1}{2} \mu_{0} M_{s}^{2}\left(N_{x} m_{x}^{2}+N_{y} m_{y}^{2}+N_{z} m_{z}^{2}\right),
$$

where $m_{x}, m_{y}$, and $m_{z}$ are the three components of the normalized magnetization $\mathbf{m}$ and $N_{x}, N_{y}$, and $N_{z}$ are the demagnetizing factors along the corresponding directions. Since $m_{x}^{2}+m_{y}^{2}+m_{z}^{2}=1$, we have

$$
\begin{aligned}
E_{\text {total }}= & \frac{1}{2} \mu_{0} M_{s}^{2}\left(N_{x}-N_{y}\right) m_{x}^{2} \\
& +\frac{1}{2} \mu_{0} M_{s}^{2}\left(N_{z}-N_{y}\right) m_{z}^{2}+\frac{1}{2} \mu_{0} M_{s}^{2} N_{y},
\end{aligned}
$$

and $\mathbf{H}_{\text {eff }}$ can be derived from the magnetic energy density of the free layer as

$$
\mathbf{H}_{\mathrm{eff}}=\left(N_{y}-N_{x}\right) M_{s} m_{x}-\left(N_{z}-N_{y}\right) M_{s} m_{z},
$$


with the easy axis anisotropy field $\mathbf{H}_{\mathbf{k}}$ in the long axis direction with strength $\left(N_{y}-N_{x}\right) M_{s}$ and the demagnetizing field $\mathbf{H}_{\mathbf{d}}$ normal to the film plane with strength $\left(N_{z}-N_{y}\right) M_{s}$.

2.2. Demagnetizing Factors. The analytic demagnetizing factors for elliptical disk can be expressed as a power series expansion of the ratio between the disk thickness and long axis length, $\tau=t_{f} / a[30,31]$, with a "squat elliptic cylinder" limit $\left(b>2 t_{f}\right)$ :

$$
\begin{aligned}
& N_{x}=\frac{1}{8 \pi^{2} \lambda}\left(J_{1}-J_{2}\right), \\
& N_{y}=\frac{\lambda}{8 \pi^{2}}\left(J_{1}+J_{2}\right), \\
& N_{z}=1-N_{x}-N_{y},
\end{aligned}
$$

where

$$
\begin{aligned}
J_{1} & =16 \tau\left\{L_{0}-\left(\frac{1}{2}+\ln \frac{\tau}{4}\right) I_{0}+\frac{\tau^{2}}{8}\left[L_{1}\right.\right. \\
& \left.\left.+\left(\frac{1}{4}-\ln \frac{\tau}{4}\right) I_{1}\right]+O\left(\tau^{4}\right)\right\}, \\
J_{2} & =\frac{16 \tau}{\gamma}\left\{(1+\varepsilon) L_{-1}-L_{0}+\left(\frac{3}{2}-\ln \frac{\tau}{4}\right)\left[(1+\varepsilon) I_{-1}\right.\right. \\
& \left.-I_{0}\right]-\frac{\tau^{2}}{24}\left[(1+\varepsilon) L_{0}-L_{1}\right. \\
& \left.\left.-\left(\frac{5}{12}+\ln \frac{\tau}{4}\right)\left[(1+\varepsilon) I_{0}-I_{1}\right]\right]+O\left(\tau^{4}\right)\right\}, \\
I_{-1} & =E\left(1-\frac{1}{\lambda^{2}}\right), \\
I_{0} & =K\left(1-\frac{1}{\lambda^{2}}\right), \\
I_{1} & =\beta^{2} K\left(1-\frac{1}{\lambda^{2}}\right), \\
L_{-1} & =\frac{1+\lambda^{2}}{2 \lambda^{2}} K\left(1-\frac{1}{\lambda^{2}}\right)-\left(\frac{\ln \lambda}{2}+1\right) E\left(1-\frac{1}{\lambda^{2}}\right), \\
L_{0} & =-\frac{\ln \lambda}{2} K\left(1-\frac{1}{\lambda^{2}}\right), \\
L_{1} & =\lambda^{2}\left[\left(1-\frac{\ln \lambda}{2}\right) E\left(1-\frac{1}{\lambda^{2}}\right)-\frac{1+\lambda^{2}}{2 \lambda^{2}} K(1\right. \\
& \left.\left.-\frac{1}{\lambda^{2}}\right)\right],
\end{aligned}
$$

with aspect ratio $\lambda=a / b, \varepsilon=\left(\lambda^{2}-1\right) /\left(\lambda^{2}+1\right)$, and $K, E$ refer to the complete elliptic integral of the first and second kind, respectively. In previous research works, simplified shape anisotropy was commonly used as $H_{k s}=2(1 / b-1 / a) t_{f} M_{s}$ [3235]. It however leads to overestimation as Figure 2 depicts. For an MTJ sample with a size of $45 \mathrm{~nm} \times 15 \mathrm{~nm} \times 3 \mathrm{~nm}$,

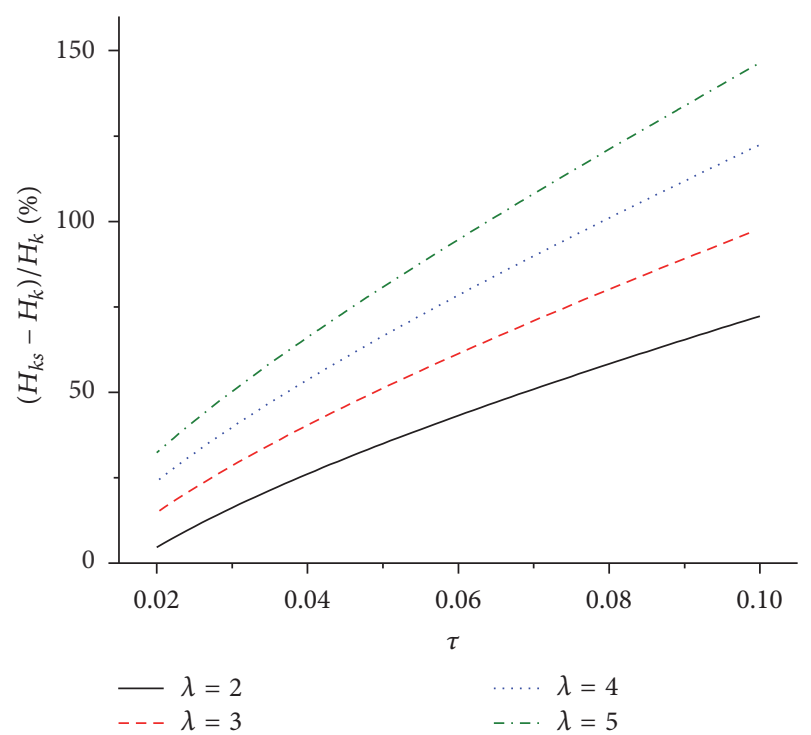

FIGURE 2: The deviation percentage caused by simplified shape anisotropy with varied $\tau$ and $\lambda$.

the shape anisotropy field is overestimated by $70 \%$, leading to poor predictions of device characteristics.

2.3. Current Density for Instability and Switching. The analytical expression of the critical current density necessary for the in-plane MTJ free layer magnetization to become unstable has been introduced by Sun [36] as follows:

$$
J_{\text {ins }}=\frac{2 e \alpha t_{f} \mu_{0} M_{s}}{\hbar \eta}\left(H_{k}+\frac{H_{d}}{2}\right) .
$$

And the critical current density for magnetization switching is given by $[37,38]$ as

$$
J_{\mathrm{sw}}=\frac{4 e \alpha t_{f} \mu_{0} M_{s}}{\pi \hbar \eta} \sqrt{H_{d}\left(H_{d}+H_{k}\right)}
$$

where $\eta$ is the spin polarization, and for SHE device it should be the effective spin Hall angle. An intermediate current density will drive the magnetization towards oscillation state, which is not sensitive to the initial angle $\theta_{0}$. With results from (6), the analytical critical current densities for an in-plane SHE-RAM can be written as

$$
\begin{aligned}
& J_{\text {ins }}=\frac{e \alpha t_{f} \mu_{0} M_{s}^{2}}{\hbar \theta_{\mathrm{SH}}^{\mathrm{eff}}}\left(1-3 N_{x}\right), \\
& J_{\mathrm{sW}}=\frac{4 e \alpha t \mu_{0} M_{s}^{2}}{\pi \hbar \theta_{\mathrm{SH}}^{\mathrm{eff}}} \sqrt{\left(N_{z}-N_{y}\right)\left(N_{z}-N_{x}\right) .}
\end{aligned}
$$

\section{Zero Temperature Simulation Results}

3.1. Analytic Results. In this section, we exclude the influence from temperature and use a free layer with $t_{f}=1.5 \mathrm{~nm}$ and $\lambda=2$. Figure 3 shows $J_{\text {ins }}$ and $J_{\text {sw }}$ dependence on the long axis length of the free layer. It can be seen that $J_{\text {sw }}$ drops more 


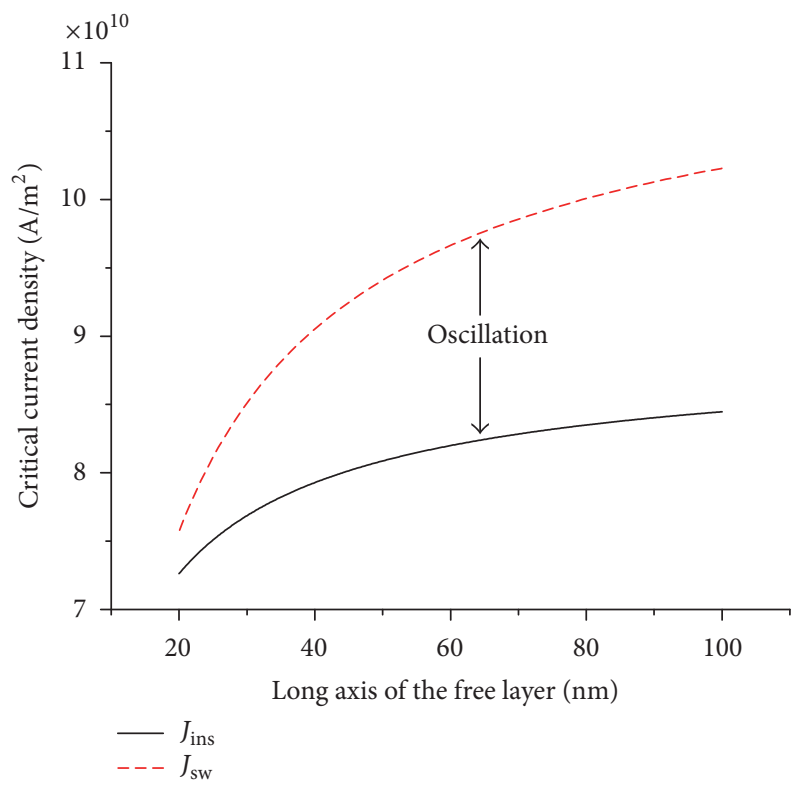

FIGURE 3: $J_{\text {ins }}$ and $J_{\text {sw }}$ dependence on long axis length of the free layer with $t_{f}=1.5 \mathrm{~nm}$ and $\lambda=2$. Intermediate current density leads to stable oscillation of $\mathbf{m}$.

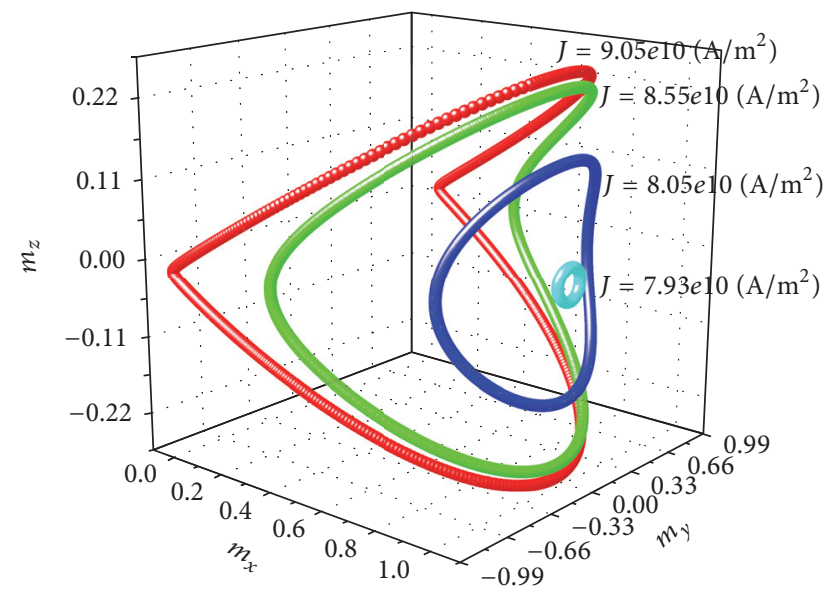

FIGURE 4: Magnetization oscillating trajectory with different current densities for in-plane free layer with initial state $m_{x} \approx 1$. Higher current density leads to oscillation with larger cone angle and finally switching. The free layer size is $40 \mathrm{~nm} \times 20 \mathrm{~nm} \times 1.5 \mathrm{~nm}$, with $J_{\text {ins }}=$ $7.93 \times 10^{10} \mathrm{~A} / \mathrm{m}^{2}$ and $J_{\text {sw }}=9.05 \times 10^{10} \mathrm{~A} / \mathrm{m}^{2}$.

rapidly and approaches $J_{\text {ins }}$ as the lateral dimension scales down, which also indicates that a smaller current density span allows for oscillation to occur. The magnetization oscillating trajectories with different current densities are shown in Figure 4. $J_{\text {ins }}$ leads to small cone angle precession illustrated by the cyan circle, while $J_{\text {sw }}$ leads to a clamshellshaped trajectory illustrated in red. Current density higher than $J_{\mathrm{sw}}$ leads to magnetization switching. The results from the analytical expressions agree with that obtained from macrospin modeling.

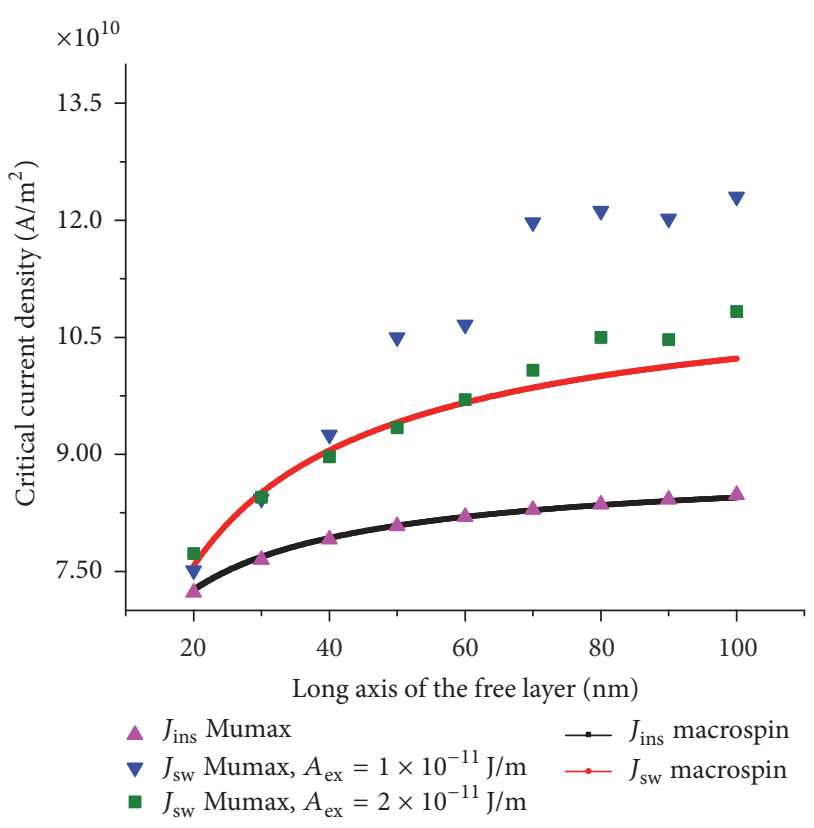

FIGURE 5: The critical current densities obtained from macrospin model and micromagnetic simulation.

3.2. Macrospin and Micromagnetic Results. The critical current densities based on macrospin and micromagnetic simulations are compared in Figure 5. An open-source code, Mumax [39], was used for micromagnetic simulation with a mesh of $64 \times 32 \times 1$ set for all geometries for better GPU performance. The initial magnetization tilt angle is 5 degrees. Good consistency can be observed in $J_{\text {ins }}$, indicating uniform magnetization for small cone angle precession. The discrepancy in $J_{\text {sw }}$ indicates that noncoherent oscillating or switching behavior takes place for larger nanomagnet size. For samples with exchange stiffness constant $A_{\text {ex }}=1 \times 10^{-11} \mathrm{~J} / \mathrm{m}, J_{\text {sw }}$ rises due to increased exchange energy when $a>40 \mathrm{~nm}$ and keeps around $1.2 \times 10^{11} \mathrm{~A} / \mathrm{m}^{2}$ when $a>70 \mathrm{~nm}$, as shown by the blue triangle. For samples with $A_{\mathrm{ex}}=2 \times 10^{-11} \mathrm{~J} / \mathrm{m}$, the discrepancy is alleviated and can be observed when $a>60 \mathrm{~nm}$, as shown by the green square.

Figure 6 shows the magnetization components during the switching process of an $80 \mathrm{~nm} \times 40 \mathrm{~nm} \times 1.5 \mathrm{~nm}$ free layer. The applied current density is $1.2 \times 10^{11} \mathrm{~A} / \mathrm{m}^{2}$. It can be seen that the magnetization experiences a period of oscillation before switching occurs. Figure 7 shows the corresponding magnetization spatial distribution. The noncoherent magnetization behavior can be observed at the two ends of the free layer, which is more obvious in Figure $7(\mathrm{~d})$. Magnetization switching occurs when the magnetization in the central part of the free layer oscillates across the short axis.

\section{Thermal Effect and Stability Analysis}

As the free layer size is scaled down below $100 \mathrm{~nm}$, the thermal fluctuation becomes significant and will have an impact on the SHE-RAM characteristics. In this section, we use (3) to account for the influence of thermal fluctuation, and 


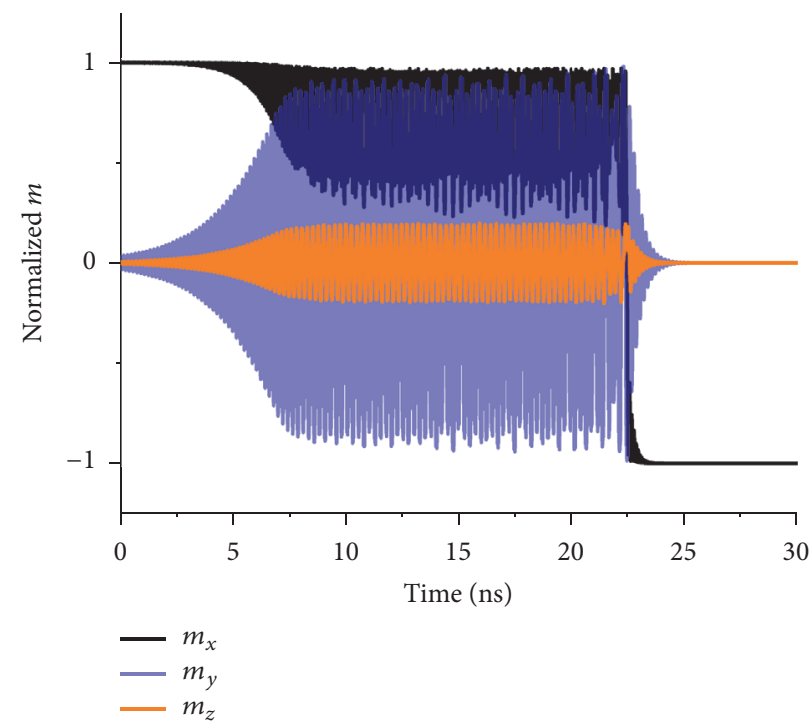

FIGURE 6: Free layer magnetization components during a switching process. The applied current density is $1.2 \times 10^{11} \mathrm{~A} / \mathrm{m}$. The magnetization oscillating time before switching is $22 \mathrm{~ns}$.

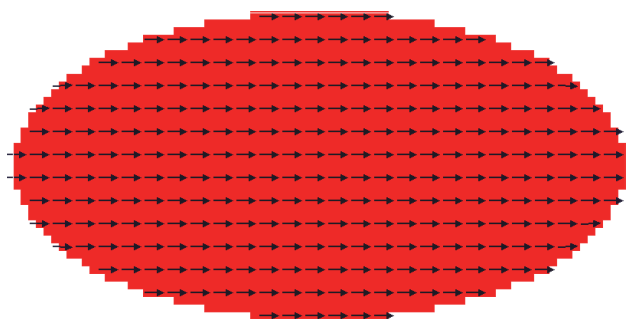

(a)

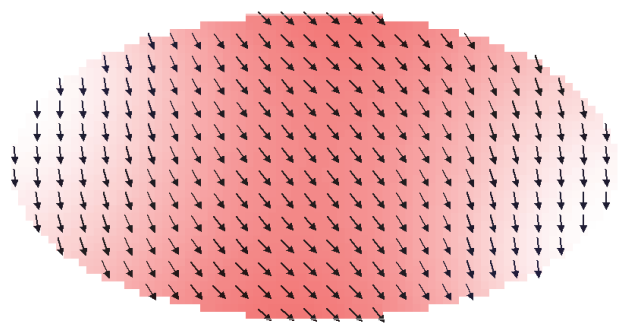

(c)

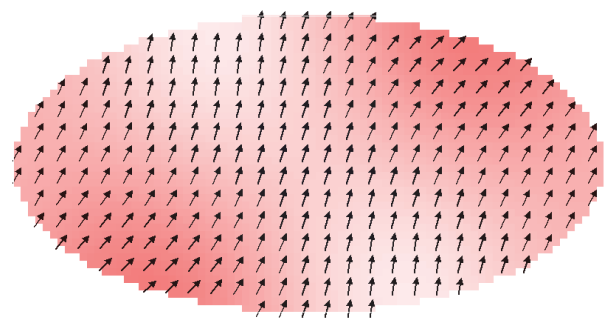

(e)

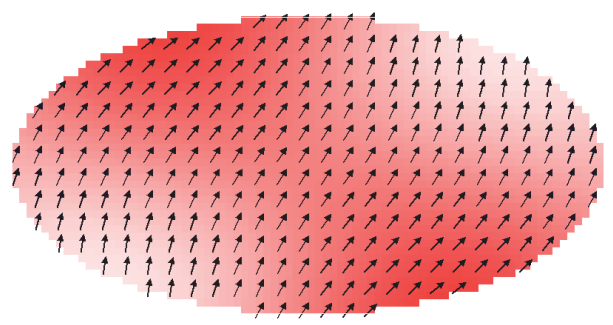

(b)

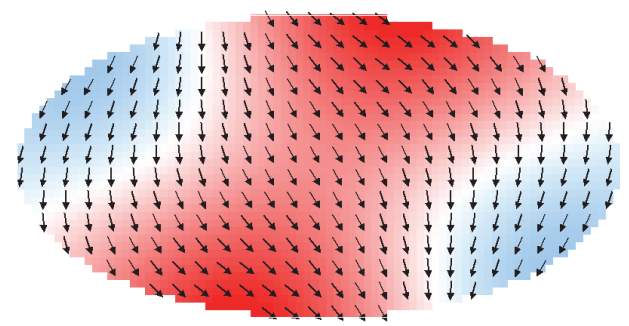

(d)

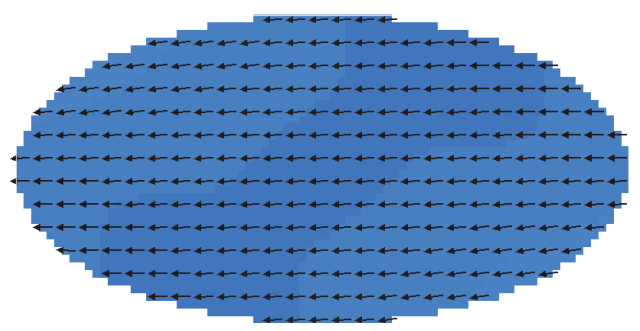

(f)

Figure 7: Magnetization spatial distribution corresponding to Figure 6. The snapshots (a) (f) are picked at 3 ns, 7 ns, 11 ns, 15 ns, 19 ns, and 23 ns during the switching process. 


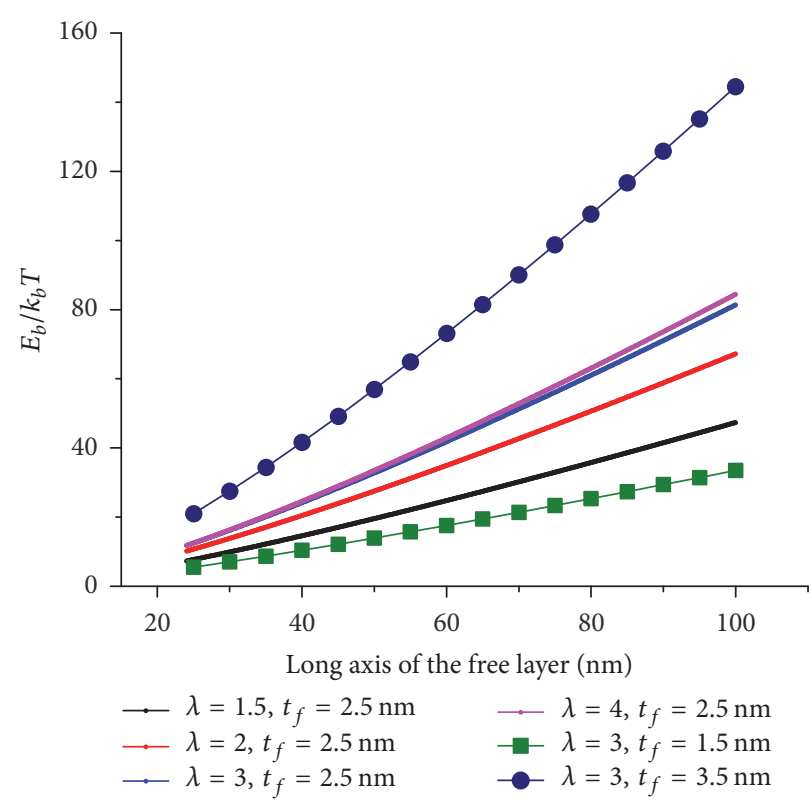

FIGURE 8: Geometric dependence of thermal stability.

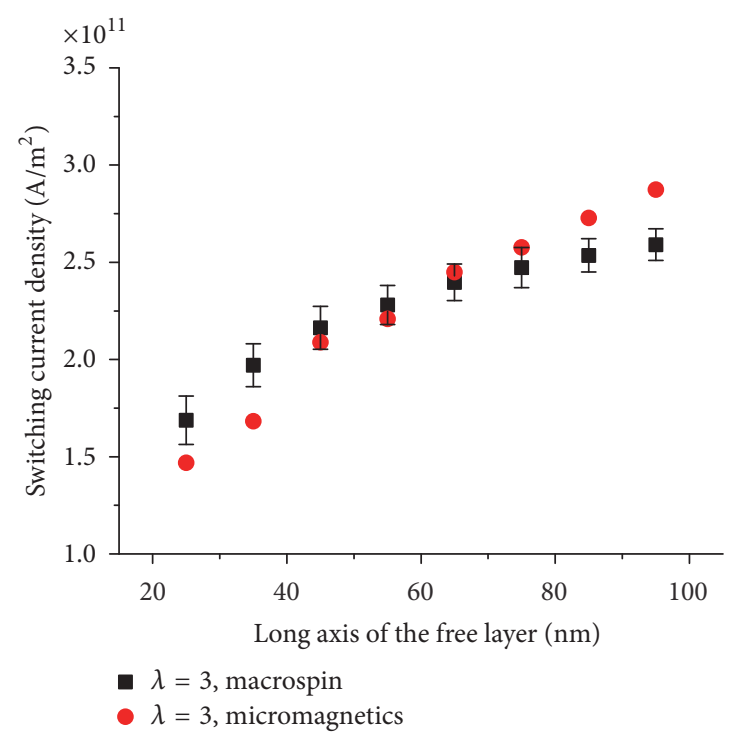

(a)

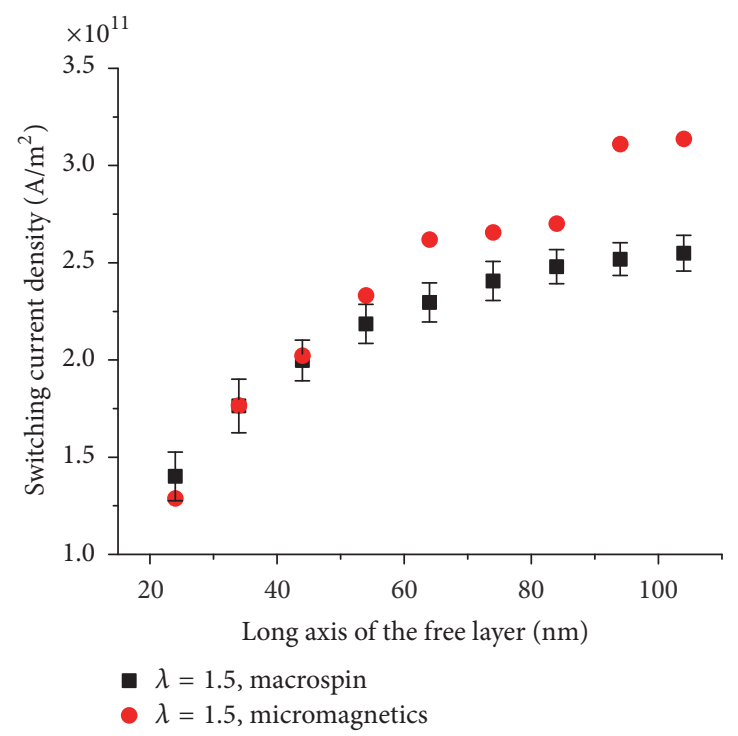

(b)

FIGURE 9: Current densities lead to magnetization switch in $5 \mathrm{~ns}$ in room temperature for free layers with (a) $\lambda=3$ and (b) $\lambda=1.5$.

the geometry dependent thermal stability and the spin torque efficiency in room temperature $(300 \mathrm{~K})$ are investigated.

The energy barrier of the free layer can be described by $E_{b}=\mu_{0} M_{s}^{2} V\left(N_{y}-N_{x}\right) / 2$. It is found that thermal stability $\Delta=E_{b} / k_{b} T$ scales almost linearly with the long axis length rather than the area of free layer, as shown in Figure 8. The reason is that the shape anisotropy which determines the energy barrier decreases with the dimension of the free layer. This tendency is similar to that of perpendicular MTJs used for conventional STT-RAM [23]. We further investigate the influences from aspect ratio $\lambda$ and free layer thickness $t_{f}$.
It is found that $\Delta$ can be effectively improved by tapering the free layer; this gain however saturates when $\lambda>3$, as illustrated by the solid lines without symbol in Figure 8 . The improvement of $\Delta$ with free layer thickness can be attributed to the enhanced easy axis anisotropy and a larger volume, as shown by lines with solid symbol.

Figure 9 shows the critical current density necessary for a free layer with $t_{f}=3.5 \mathrm{~nm}$ to switch in $5 \mathrm{~ns}$ in room temperature. The results from both macrospin and micromagnetic modeling are shown. It can be noted that, despite the improvement in thermal stability, higher $\lambda$ does not lead to an 


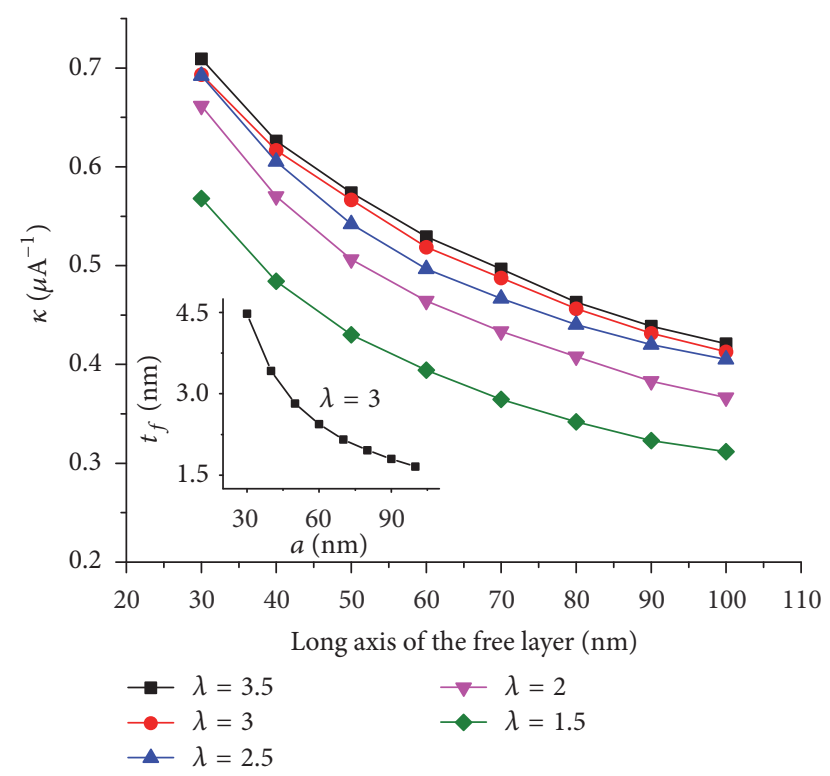

FIGURE 10: Geometric dependence of spin torque switching efficiency with fixed thermal stability $\Delta=40$. The inset shows the corresponding adjustment of thickness of free layer with $\lambda=3$.

obvious increase in switching current density, which is due to a smaller demagnetizing field. We used $A_{\mathrm{ex}}=1 \times 10^{-11} \mathrm{~J} / \mathrm{m}$ for micromagnetic modeling. The discrepancy between results from macrospin and micromagnetic modeling for larger geometries indicates the nonuniform magnetization during switching process that, for smaller geometries, as shown in Figure 9(a), might be caused by coarse mesh. It can be noted that the free layer with $\lambda=1.5$ is more susceptible to nonuniform behavior, and this is due to a larger area compared with free layers with $\lambda=3$.

The spin torque efficiency is defined as $\kappa=\Delta / I_{c}$. For SHERAM, $I_{c}=J_{c} a t_{w}$, where $t_{w}$ is the thickness of the tungsten layer. In order to analyze the geometric dependence of $\kappa$ for devices satisfying the retention requirement, the thermal stability $\Delta$ is fixed at 40 by adjusting the thickness of the free layer, while the length of long axis, $a$, and the aspect ratio, $\lambda$, are varied. It is found that the spin torque efficiency decreases with $a$, as shown in Figure 10. Moreover, improvement of $\kappa$ can be observed in the free layer with higher aspect ratio. This improvement also saturates when $\lambda>3$, similar to that of thermal stability. The inset of Figure 10 shows the thickness adjustment of free layer with $\lambda=3$.

\section{Conclusion}

In this paper, we have studied the influence of shape anisotropy on switching dynamics of elliptic nanomagnets by introducing accurate demagnetizing factors and theoretically investigated the characteristics of SHE-RAM with in-plane magnetization. Analytic expressions of the key performance indicators for in-plane SHE-RAM are obtained including the critical switching current density, thermal stability, and spin torque efficiency, which are found to be highly geometry dependent. In particular, the thermal stability and spin torque efficiency can be effectively improved by selecting the appropriate aspect ratio of the free layer. Moreover, the spin torque efficiency tends to increase as the SHE-RAM element is scaled down. The demagnetizing factors can also be applied to the macrospin approach to give more reliable characteristic predictions for sub-100 $\mathrm{nm}$ spin torque based device.

\section{Competing Interests}

The authors declare that they have no competing interests.

\section{Acknowledgments}

This work was supported by the National Natural Science Foundation of China (Grant no. 61274089) and the International Scientific and Technological Cooperation Project of Shanxi Province, China (2014081029-2).

\section{References}

[1] J. C. Slonczewski, "Current-driven excitation of magnetic multilayers," Journal of Magnetism and Magnetic Materials, vol. 159, no. 1-2, pp. L1-L7, 1996.

[2] L. Berger, "Emission of spin waves by a magnetic multilayer traversed by a current," Physical Review B-Condensed Matter and Materials Physics, vol. 54, no. 13, pp. 9353-9358, 1996.

[3] D. C. Ralph and M. D. Stiles, "Spin transfer torques," Journal of Magnetism and Magnetic Materials, vol. 320, no. 7, pp. 1190-1216, 2008.

[4] K. L. Wang, J. G. Alzate, and P. K. Amiri, "Low-power nonvolatile spintronic memory: STT-RAM and beyond," Journal of Physics D: Applied Physics, vol. 46, no. 8, Article ID 074003, 2013.

[5] S. A. Wolf, D. D. Awschalom, R. A. Buhrman et al., "Spintronics: A spin-based electronics vision for the future," Science, vol. 294, no. 5546, pp. 1488-1495, 2001.

[6] T. Kawahara, K. Ito, R. Takemura, and H. Ohno, "Spin-transfer torque RAM technology: review and prospect," Microelectronics Reliability, vol. 52, no. 4, pp. 613-627, 2012.

[7] J. A. Katine and E. E. Fullerton, "Device implications of spintransfer torques," Journal of Magnetism and Magnetic Materials, vol. 320, no. 7, pp. 1217-1226, 2008.

[8] Z. Duan, A. Smith, L. Yang et al., "Nanowire spin torque oscillator driven by spin orbit torques," Nature Communications, vol. 5, article no. 5616, 2014.

[9] D. Houssameddine, U. Ebels, B. Delaët et al., "Spin-torque oscillator using a perpendicular polarizer and a planar free layer," Nature Materials, vol. 6, no. 6, pp. 447-453, 2007.

[10] T. Chen, R. K. Dumas, A. Eklund et al., "Spin-torque and spinhall nano-oscillators," https://arxiv.org/abs/1512.03162.

[11] J. Kim, A. Paul, P. A. Crowell et al., "Spin-based computing: device concepts, current status, and a case study on a highperformance microprocessor," Proceedings of the IEEE, vol. 103, no. 1, pp. 106-130, 2015.

[12] S. Mangin, D. Ravelosona, J. A. Katine, M. J. Carey, B. D. Terris, and E. E. Fullerton, "Current-induced magnetization reversal in nanopillars with perpendicular anisotropy," Nature Materials, vol. 5, no. 3, pp. 210-215, 2006.

[13] S. Mangin, Y. Henry, D. Ravelosona, J. A. Katine, and E. E. Fullerton, "Reducing the critical current for spin-transfer 
switching of perpendicularly magnetized nanomagnets," Applied Physics Letters, vol. 94, no. 1, Article ID 012502, 2009.

[14] S. Ikeda, K. Miura, H. Yamamoto et al., "A perpendicularanisotropy $\mathrm{CoFeB}-\mathrm{MgO}$ magnetic tunnel junction," Nature Materials, vol. 9, no. 9, pp. 721-724, 2010.

[15] W. Zhao, X. Zhao, B. Zhang et al., "Failure analysis in magnetic tunnel junction nanopillar with interfacial perpendicular magnetic anisotropy," Materials, vol. 9, no. 1, article 41, 2016.

[16] J. Sinova, S. O. Valenzuela, J. Wunderlich, C. H. Back, and T. Jungwirth, "Spin Hall effects," Reviews of Modern Physics, vol. 87, no. 4, pp. 1213-1260, 2015.

[17] Y. Seo, X. Fong, K.-W. Kwon, and K. Roy, "Spin-hall magnetic random-access memory with dual read/write ports for on-chip caches," IEEE Magnetics Letters, vol. 6, pp. 1-4, 2015.

[18] S. Manipatruni, D. E. Nikonov, and I. A. Young, "Energydelay performance of giant spin Hall effect switching for dense magnetic memory," Applied Physics Express, vol. 7, no. 10, Article ID 103001, 2014.

[19] C.-F. Pai, L. Liu, Y. Li, H. W. Tseng, D. C. Ralph, and R. A. Buhrman, "Spin transfer torque devices utilizing the giant spin Hall effect of tungsten," Applied Physics Letters, vol. 101, no. 12, Article ID 122404, 2012.

[20] Q. Hao and G. Xiao, "Giant spin Hall effect and switching induced by spin-transfer torque in a W/ $\mathrm{Co}_{40} \mathrm{Fe}_{40} \quad \mathrm{~B}_{20} / \mathrm{MgO}$ structure with perpendicular magnetic anisotropy," Physical Review Applied, vol. 3, no. 3, Article ID 034009, 2015.

[21] L. Liu, C.-F. Pai, Y. Li, H. W. Tseng, D. C. Ralph, and R. A. Buhrman, "Spin-torque switching with the giant spin hall effect of tantalum," Science, vol. 336, no. 6081, pp. 555-558, 2012.

[22] I. M. Miron, K. Garello, G. Gaudin et al., "Perpendicular switching of a single ferromagnetic layer induced by in-plane current injection,” Nature, vol. 476, no. 7359, pp. 189-193, 2011.

[23] J. Z. Sun, S. L. Brown, W. Chen et al., "Spin-torque switching efficiency in CoFeB-MgO based tunnel junctions," Physical Review B-Condensed Matter and Materials Physics, vol. 88, no. 10, Article ID 104426, 2013.

[24] H. Sato, E. C. I. Enobio, M. Yamanouchi et al., "Properties of magnetic tunnel junctions with a $\mathrm{MgO} / \mathrm{CoFeB} / \mathrm{Ta} / \mathrm{CoFeB} / \mathrm{MgO}$ recording structure down to junction diameter of $11 \mathrm{~nm}$," Applied Physics Letters, vol. 105, no. 6, Article ID 062403, 2014.

[25] M. Gajek, J. J. Nowak, J. Z. Sun et al., "Spin torque switching of $20 \mathrm{~nm}$ magnetic tunnel junctions with perpendicular anisotropy," Applied Physics Letters, vol. 100, no. 13, Article ID 132408, 2012.

[26] G. D. Chaves-O’Flynn, G. Wolf, J. Z. Sun, and A. D. Kent, "Thermal stability of magnetic states in circular thin-film nanomagnets with large perpendicular magnetic anisotropy," Physical Review Applied, vol. 4, no. 2, Article ID 024010, 2015.

[27] G. Finocchio, M. Carpentieri, E. Martinez, and B. Azzerboni, "Switching of a single ferromagnetic layer driven by spin Hall effect," Applied Physics Letters, vol. 102, no. 21, Article ID 212410, 2013.

[28] S. Fukami, T. Anekawa, C. Zhang, and H. Ohno, "A spin-orbit torque switching scheme with collinear magnetic easy axis and current configuration," Nature Nanotechnology, vol. 11, pp. 621625, 2016.

[29] W. F. Brown Jr., "Thermal fluctuations of a single-domain particle," Journal of Applied Physics, vol. 34, no. 4, pp. 1319-1320, 1963.

[30] D. A. Goode and G. Rowlands, "The demagnetizing energies of a uniformly magnetized cylinder with an elliptic cross-section,"
Journal of Magnetism and Magnetic Materials, vol. 267, no. 3, pp. 373-385, 2003.

[31] M. Beleggia, M. De Graef, Y. T. Millev, D. A. Goode, and G. Rowlands, "Demagnetization factors for elliptic cylinders," Journal of Physics D: Applied Physics, vol. 38, no. 18, pp. 33333342,2005 .

[32] N. D. Rizzo, D. Houssameddine, J. Janesky et al., "A fully functional $64 \mathrm{Mb}$ DDR3 ST-MRAM built on $90 \mathrm{~nm}$ CMOS technology," IEEE Transactions on Magnetics, vol. 49, no. 7, pp. 4441-4446, 2013.

[33] R. Dorrance, F. Ren, Y. Toriyama, A. A. Hafez, C.-K. K. Yang, and D. Marković, "Scalability and design-space analysis of a 1T-1MTJ memory cell for STT-RAMs," IEEE Transactions on Electron Devices, vol. 59, no. 4, pp. 878-887, 2012.

[34] K. C. Chun, H. Zhao, J. D. Harms, T.-H. Kim, J.-P. Wang, and C. H. Kim, "A scaling roadmap and performance evaluation of in-plane and perpendicular MTJ based STT-MRAMs for highdensity cache memory," IEEE Journal of Solid-State Circuits, vol. 48, no. 2, pp. 598-610, 2013.

[35] D. Apalkov, S. Watts, A. Driskill-Smith, E. Chen, Z. Diao, and V. Nikitin, "Comparison of scaling of in-plane and perpendicular spin transfer switching technologies by micromagnetic simulation," IEEE Transactions on Magnetics, vol. 46, no. 6, pp. 2240 2243, 2010.

[36] J. Z. Sun, "Spin-current interaction with a monodomain magnetic body: A Model Study," Physical Review B-Condensed Matter and Materials Physics, vol. 62, no. 1, pp. 570-578, 2000.

[37] D. Pinna, A. D. Kent, and D. L. Stein, “Thermally assisted spintransfer torque dynamics in energy space," Physical Review BCondensed Matter and Materials Physics, vol. 88, no. 10, Article ID 104405, 2013.

[38] T. Taniguchi, Y. Utsumi, M. Marthaler, D. S. Golubev, and H. Imamura, "Spin torque switching of an in-plane magnetized system in a thermally activated region," Physical Review BCondensed Matter and Materials Physics, vol. 87, no. 5, Article ID 054406, 2013.

[39] A. Vansteenkiste, J. Leliaert, M. Dvornik, M. Helsen, F. GarciaSanchez, and B. Van Waeyenberge, "The design and verification of MuMax3," AIP Advances, vol. 4, no. 10, Article ID 107133, 2014. 

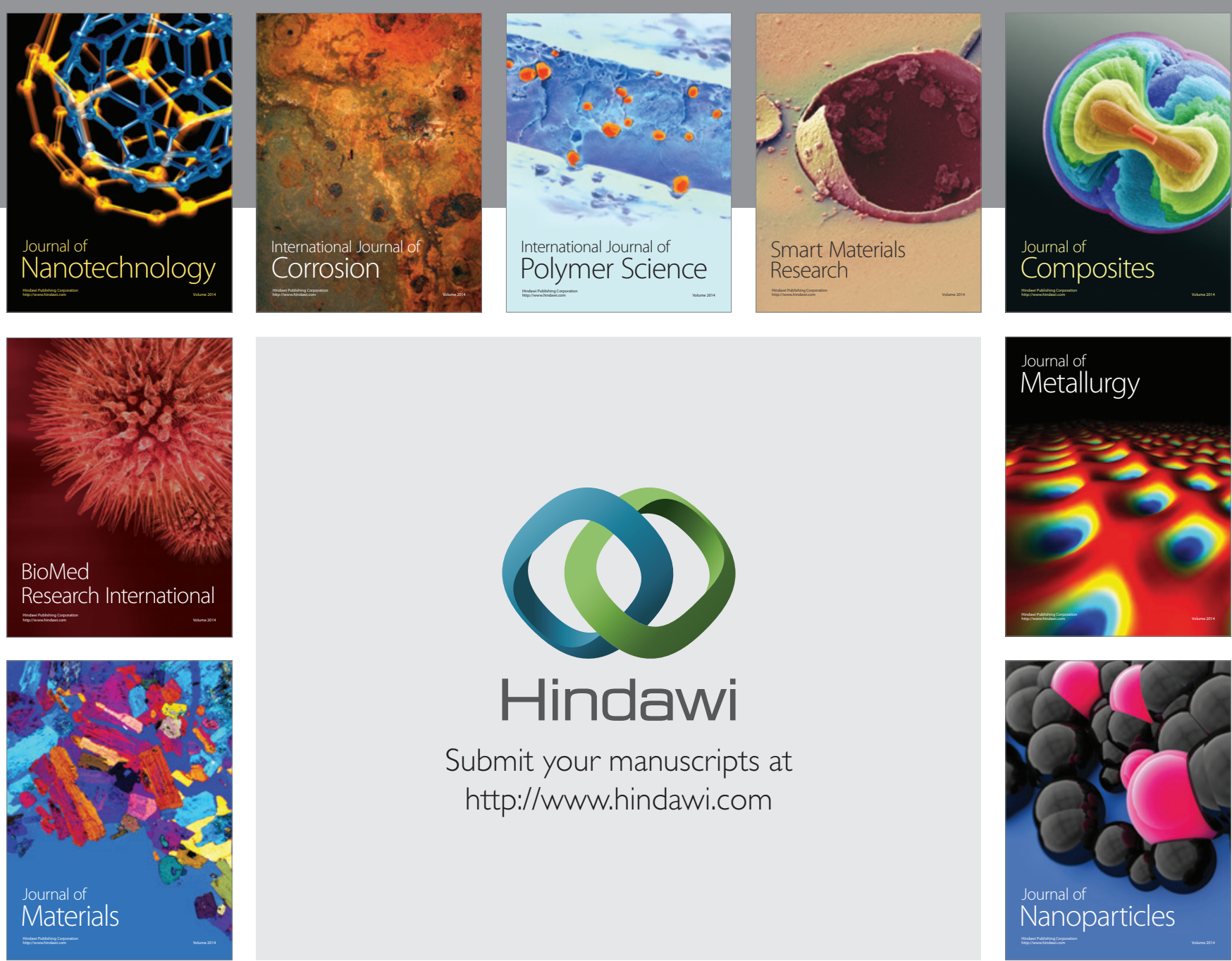

\section{Hindawi}

Submit your manuscripts at

http://www.hindawi.com

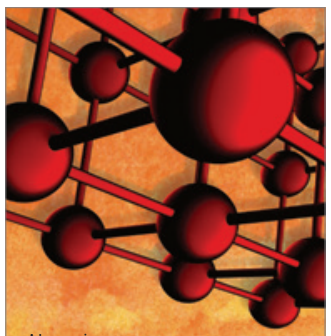

Materials Science and Engineering
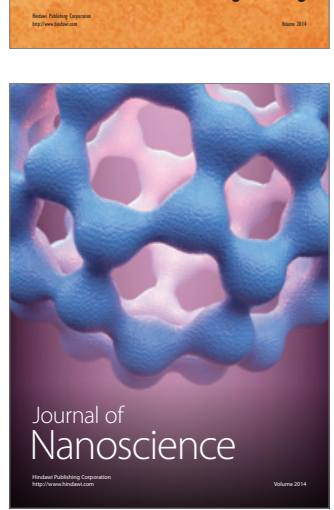
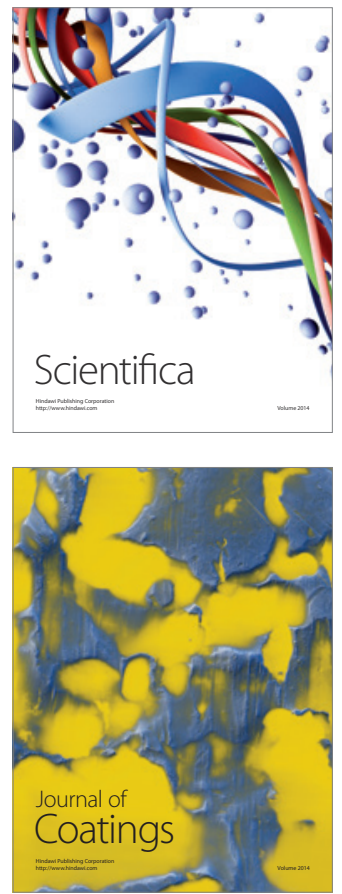
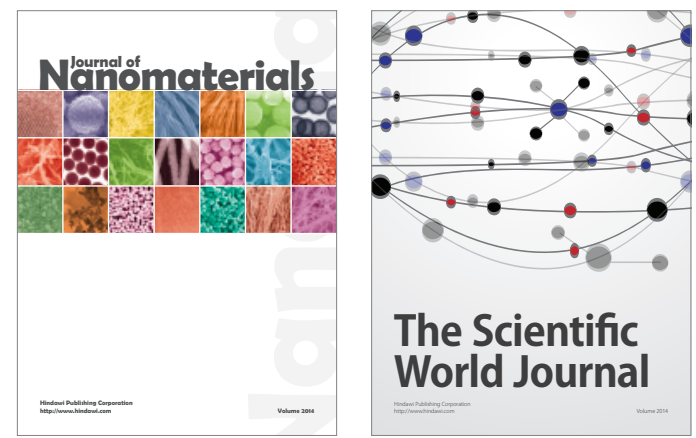

The Scientific World Journal
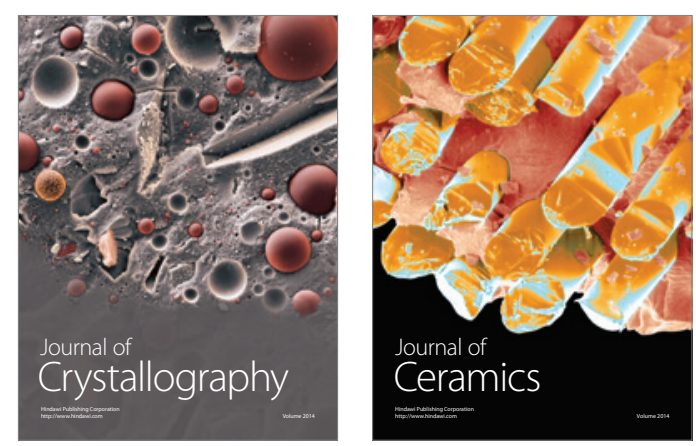
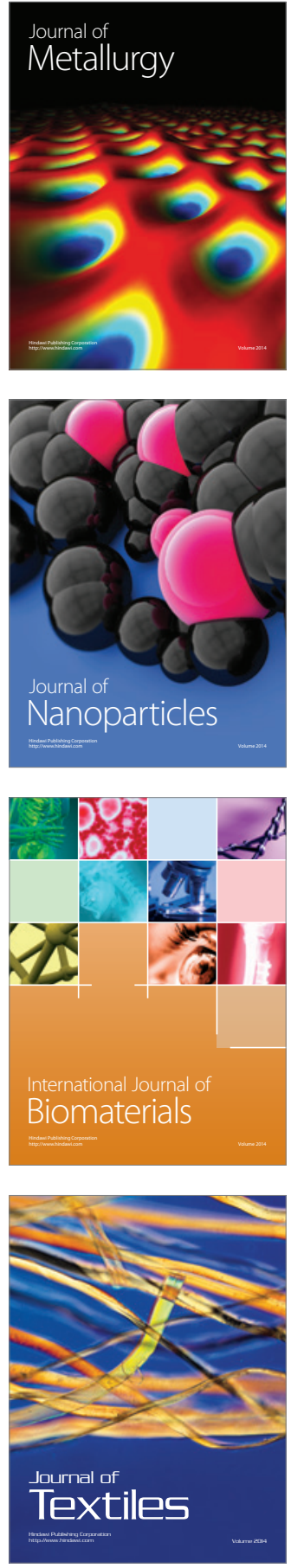